\title{
Pattern of Specific Dermatoses During Pregnancy
}

\author{
Afroz $\mathrm{R}^{1}$, Chowdhury $\mathrm{SA}^{2}$, Mannan $\mathrm{MA}^{3}$, Talukdar $\mathrm{SK}^{4}$
}

\begin{abstract}
Dermatoses unique to pregnancy are rare but important to recognize because they may be intensely pruritic or painful to the mother and they may pose significant risk to the patient, her fetus or both. Dermatoses of pregnancy usually does not get attention. Both the obstetrician and the patient are more focused on the pregnancy itself. Some dermatoses of pregnancy elevate the risk of adverse fetal events, it is critical to distinguish them from underlying skin conditions that simply present or flare during pregnancy. Early diagnosis and treatment may help to reduce morbidity and mortality to the gravid patient and her fetus and minimize fetal exposure to unnecessary treatments.
\end{abstract}

This clinical study shows, about 8\% pregnant women had specific dermatoses. Maximum $40 \quad$ (41.6\%) patients were in between the age of 25 to 29 years. According to trimester, $60(62.5 \%)$ patients were in $3 r d$ trimester. In case of gravida, maximum 59 (61.5\%) patients were primigravida. In this study, pruritic urticarial papules and plaques of pregnancy ( PUPPP) was the most common dermatoses during pregnancy.

Key words: pruritic urticarial papules and plaques of pregnancy, pruritic Folliculitis of pregnancy, prurigo gravidarum, papular dermatitis of pregnancy, prurigo of pregnancy (Besnier), herpes gestationis and impetigo herpetiformis.

\section{Introduction}

Pregnancy is a special time for a woman. Women experience significant endocrine and metabolic changes during pregnancy which are capable of causing both physiologic and pathologic alteration in skin, nail and

1. Corresponding Author: Dr. Rozina Afroz Registrar, Skin \& VD Department Shaheed Ziaur Rahman Medical College, Bogra.

2. Dr. Shahabuddin Ahmed Chowdhury Associated Professor \& Head Skin \& VD Department Shaheed Ziaur Rahman Medical College, Bogra.

3. Dr. M.A. Mannan Assistant Professor Skin \& VD Department Shaheed Ziaur Rahman Medical College, Bogra.

4. Dr. Sudarshan Kumar Talukdar Assistant Registrar Skin \& VD Department Shaheed Ziaur Rahman Medical College, Bogra. hair. The majority of physiologic skin conditions related to gestation resolve after child birth ${ }^{1}$. The specific dermatoses of pregnancy is unique to gestation. There are challenges to diagnosing pregnancy related dermatoses. Most of these dermatoses are poorly characterized and poorly understood. There is significant overlap between their clinical features and only nonspecific laboratory findings may exist to support their diagnosis. Clinicians familiar with the cutaneous manifestations of pregnancy can most effectively treat and counsel patients, guide expectations and avoid unnecessary diagnostic tests and therapies ${ }^{2}$. Awareness of pregnancy related skin changes can facilitate improved care of woman during pregnancy by identifying those skin changes that require further evaluation, effect of pregnancy on preexisting skin diseases, alerting the attending obstetrician any possible complication and adverse outcome on fetus or mother during antenatal, prenatal, postnatal period and possible risk of recurrence in subsequent pregnancies ${ }^{3}$.

There are three general categories of pregnancy associated skin conditions ${ }^{1}$. Physiological skin change in pregnancy ${ }^{2}$ preexisting skin conditions that change during pregnancy ${ }^{3}$ Pregnancy specific dermatoses ${ }^{4}$. Hormonal changes cause hyperpigmentation in most of the women during pregnancy. The areolae, axillae \& genitals are most commonly affected. Melasma (chloasma) may be the most cosmetically troublesome skin condition associated with pregnancy. Vascular change is common in pregnancy which usually resolves in the first few months after pregnancy. Other changes like spider angiomas,varicosities, granuloma gravidarum, nonpitting oedema of face, eyelids and extremities, pyogenic granuloma, vascular instability. e.g facial flushing, pallor, cutis marmorata of the legs and Raynaud's phenomenon. Glandular changes include increase function of eccrine gland resulting in sebaceous gland hyper activity and hypertrophy causes acne. Striae gravidarum occur in up to 90 percent of pregnant woman by third trimester. Striae most commonly appear on abdomen but may appear on breast, thigh and inguinal area. The cause of striae is multifactorial and includes physical factors (e.g actual stretching of the skin) and hormonal factors (e.g effects of adrenocortical steroids, estrogen and relaxin on the skin's elastic fibers ${ }^{5}$.

Amongst skin conditions influenced by pregnancy, certain skin conditions like atopic dermatitis are most likely to 
ORIGINAL ARTICLE

worsen or appear for first time during pregnancy or post-partum ${ }^{6}$. Eczema has a fluctuating course in most patients and is influenced environmental and internal triggers. However, Pregnancy does seem to have an effect on eczema in most women with condition. Pre-existing eczema may deteriorate at any stage of pregnancy, and a slightly high rate is seen in the second trimester ${ }^{7}$. Psoriasis vulgaris more likely improves with pregnancy but impetigo herpetiformis which is generalized pustular psoriasis is associated with pregnancy ${ }^{8}$ Systemic Lupus erythematosus (SLE) if stable for 3 months prior to conception is well tolerated but if active at conception, $50 \%$ will worsen. In Systemic Sclerosis pregnancy is relatively unaffected ${ }^{9}$.

The study was designed to assess the frequency of specific dermatoses during pregnancy. The key rationale for conducting the study was to have a better understanding of these dermatoses.

\section{Materials \& Methods}

A clinical study was carried out to find out frequency \& common variety of specific dermatoses during pregnancy. 96 patients were selected according to inclusion \& exclusion criteria in the outpatient department of Gynae and Obs in Shaheed Ziaur Rahman Medical College Hospital, Bogra. The study was carried out from June 2012 to November 2012. An informed consent was taken from each patient before entry in the study.

Data were collected on pre- designed data sheet. A detailed history and clinical examination was done for each patient.

\section{Results}

Ninety six pregnant women had been suffering from specific dermatoses. So in this observation, about $8 \%$ pregnant women had specific dermatoses. Maximum $40(7.5 \%)$ patients were in between the age of 25-29 years followed by $27(10.8 \%)$ patients in 30-34 years, $16(5.7 \%)$ patients in 20-24 years and $13(8.7 \%)$ patients were in between the age of 35-40 years.

Table-1: Distribution of specific dermatoses by age $(n=96)$

Out of 1210 case 96 patients had specific dermatoses $(7.9 \%)$

\begin{tabular}{llc}
\hline Age group & $\begin{array}{l}\text { Number } \\
\text { of patient }\end{array}$ & $\%$ \\
\hline $20-24$ & 16 & $16.6 \%$ \\
$25-29$ & 40 & $41.6 \%$ \\
$30-34$ & 27 & $28.2 \%$ \\
$35-40$ & 13 & $13.6 \%$ \\
Total & 96 & $100.0 \%$ \\
\hline
\end{tabular}

According to trimester, $60(62.5 \%)$ patients were in third trimester followed by $32(33.33 \%)$ patients were in second trimester and 4(4.2\%) patients in first trimester.
Table-2: Distribution of specific dermatoses by Trimester $(n=96)$

\begin{tabular}{clllllc}
\hline Trimester & \multicolumn{7}{c}{ Name of the disease } \\
\hline & PG & PUPPP PG of Besiner & PFP & PD & All disease \\
\hline 1st (0-12 week) & 0 & 0 & 0 & 4 & 0 & 4 \\
& $0.0 \%$ & $0.0 \%$ & $0.0 \%$ & $16.0 \%$ & $0.0 \%$ & $4.2 \%$ \\
2nd (13-28 week) & 2 & 9 & 3 & 16 & 2 & 32 \\
& $11.1 \%$ & $20.0 \%$ & $60.0 \%$ & $64.0 \%$ & $66.7 \%$ & $33.3 \%$ \\
3rd (29-40 week) & 16 & 36 & 2 & 5 & 1 & 60 \\
& $88.9 \%$ & $80.0 \%$ & $40.0 \%$ & $20.0 \%$ & $33.3 \%$ & $62.5 \%$ \\
Total & 18 & 45 & 5 & 25 & 3 & 96 \\
\hline
\end{tabular}

In case of gravida, maximum $59(61.5 \%)$ patients were primigravida and $37(38.5 \%)$ patients were multigravida.

Table-3: Distribution of specific Dermatoses by Gravida $(n=96)$

\begin{tabular}{lllllll} 
Gravida & \multicolumn{6}{l}{ Name of the disease } \\
& PG & PUPPP & PG of Besiner & PFP & PD & $\begin{array}{l}\text { All } \\
\text { disease }\end{array}$ \\
\hline \multirow{2}{*}{ Primigravida } & 6 & 34 & 3 & 14 & 2 & 59 \\
\cline { 2 - 7 } & $33.3 \%$ & $75.6 \%$ & $60.0 \%$ & $56.0 \%$ & $66.7 \%$ & $61.5 \%$ \\
\hline \multirow{2}{*}{ Multigravida } & 12 & 11 & 2 & 11 & 1 & 37 \\
\hline Total & $66.7 \%$ & $24.4 \%$ & $40.0 \%$ & $44.0 \%$ & $33.3 \%$ & $38.5 \%$ \\
\hline
\end{tabular}

In this study, Pruritic Urticarial Papules and Plaques of Pregnancy(PUPPP) was the most common dermatoses. In case of PUPPP, about $45(46.08 \%)$ patients were suffering from PUPPP. According to age distribution in PUPPP $25(55.6 \%)$ patients in between the age of 25-29 years followed by $10(22.2 \%)$ in between age of 30-34 years, 6 $(13.3 \%)$ patients in 20-24 years and $4(8.9 \%)$ patients in between age of 35-40 years. In case of trimester, $36(80 \%)$ patients in third trimester and $9(20 \%)$ in second trimester. According to gravida, maximum 34 (75.6\%) patients were primigravida and $11(24.4 \%)$ patients were multigravida. In case of Pruritic Folliculitis of Pregnancy (PFP) 25 (26.00\%) patients were suffering from this disease and 12 (48\%) patients in 25-29 years, $9(36 \%)$ patients in $30-34$ years, $4(16 \%)$ patients in 20-24 years and $16(64 \%)$ patients were in second trimester, 5(20\%) patients in third trimester, 4(16\%) in first trimester and 14(56\%) patients were primigravida, $11(44 \%)$ multigravida. In case of Prurigo Gravidarum ( Intrahepatic Cholestasis of Pregnancy) about 18 (18.75\%) patients were suffering from this disease. In ICP, maximum $8(44.4 \%)$ patients in between the age of 35-40 years followed by $4(22.2 \%)$ in $30-34$ years, $4(22.2 \%)$ in $25-29$ years, $2(11.1 \%)$ patients in 20-24 years and $16(88.9 \%)$ were in third trimester, 2 (11.1\%) patients were in second trimester and $12(66.7 \%)$ patients were multigravida, 6(33.3\%) patients were primigravida. In case of Prurigo of Pregnancy (Besnier), $5(5.20 \%)$ patients were in this disease and $2(40 \%)$ patients in 20-24 years, $2(40 \%)$ in $30-34$ years, $1(20 \%)$ in $25-29$ years and $3(60 \%)$ in second trimester, 2(40\%) in third trimester and $3(60 \%)$ were primigravida, 2(40\%) were multigravida. In case of Papular Dermatitis of Pregnancy (PD) 3 (3.12\%) 
patients were suffering from this disease and $2(66.7 \%)$ in between age of 25-29 years, 1 (33.3\%) in 20-24 years and $2(66.7 \%)$ in second trimester, $1(33.3 \%)$ in third trimester and 2(66.7\%) were primigravida, 1 (33.3\%) was multigravida. But in this observation, no case of herpes gestationis and impetigo herpetiformis was found.

Table-4: Frequency of Specific Dermatomes during pregnancy $(\mathrm{n}=96)$

\begin{tabular}{ccc}
\hline Name of the disease & Number of patient & Percentage \\
\hline PUPPP & 45 & $46.08 \%$ \\
PFP & 25 & $26 \%$ \\
ICP & 18 & $18.75 \%$ \\
PP & 5 & $5.20 \%$ \\
\hline
\end{tabular}

\section{Discussion}

A total of 96 patients were included in this study according to inclusion \& exclusion criteria. The focus of my study was on specific dermatoses during pregnancy and did not address other physiological skin conditions. Among the pregnant woman, $8 \%$ were suffering from specific dermatoses, where maximum patients were in between the age of 25-29 years. Study shows, most of the specific dermatoses occurred in the third trimester \& the incidence was common in primigravida. These findings are similar of the study done by Ambros CM March on, 20063.

In this study, PUPPP was the most common (46.08\%) dermatoses during pregnancy, followed by PFP (26\%), ICP (18.75\%), PP (5.20\%) and PD (3.12\%). These findings are mostly similar in accordance with the observations of Kroumpouzos et al4 USA10.

Women experience significant endocrine and metabolic changes during pregnancy which are capable of causing both physiologic and pathologic alterations in skin, nail \& hair. Rarer and more concerning are the specific dermatoses of pregnancy- pathological skin eruptions unique to gestation. Early diagnosis and treatment may help to reduce morbidity and mortality to the gravid patient and her fetus and minimize fetal exposure to unnecessary treatments. It is a research priority to estimate the percentage of dermatoses specific to pregnancy. The present study will help our physicians as well as our future researchers.

\section{References}

1. Wong Rc. physiologic skin changes in pregnancy. Harahapm, wallach Rc, eds. In: Skin changes and Diseases of pregnancy. New Your: Marcel Dekker, Inc, 1996: 37.

2. Mare Tunzi, Gary R, Gary. Common skin conditions during pregnancy. Am Famphysician, 2007;75: 211-8.

3. Ambros-Rudolph CM, Mullegger RR, Vaughan-Jones SA,et al. The specific dermatoses of pregnancy revisited and reclassified: Result of a retrospective two center study on 505 pregnant patients . J Am Acad Dermatol 2006;56:395-404.

4. Ioannis Koutroulis, John papoutsis. George kroumpouzos, Atopic dermatitis in pregnancy: current status and. Challenges, obstet Gynecol survey 2011;6610: 654- 663 .

5. Sophie weather head, steven E Robson, Niek J Rejnulds Eczema in pregnanacy. BMJ, 2007; 335: 152-154.

6. Elizabeth J. Horn, Chsistina D. Chambers, Alan menter. Pregnancy out comes in prosiasis, J. Am Aead Dermatol. 2009;61:5-8

7. Winton GB, Lewis CW. Dermatoses of pregnancy. J. Am Dermatol 1982; 6:977-998

8. Christina M. Ambros Rudolph, Dermatoses of pregnancy- Clues to Diagnosis, Fetal Risk and Therapy. Ann Dermatol 2011; 233: 265-275

9. Jenkins RE, Black MM. Pemphigoid (herpes) gestationis. In: Black MM, Ambros-Rudolph CM, Edwards L, Lynch P,editors. Obstetric and gynecologic dermatology. 3rd ed.London: Elsevier Limited, 2008:37-47

10. Kroumpouzos G, Cohen LM . Specific Dermatoses of pregnancy, an evidence-based systemic review. Am J obs Gynecol 2003; 188: 1083-92 